\title{
La cultura de la confianza en tiempos de crisis: análisis de los discursos
}

\section{The Culture of Trust in Times of Crisis: a discourse analysis}

\author{
*Javier Callejo \\ Instituto Complutense de Sociología para el Estudio de las Transformaciones Sociales \\ Contemporáneas (TRANSOC) \\ Facultadde CienciasPolíticasySociología.Universidad Nacionalde Educacióna Distancia. España/Spain. \\ mcallejo@poli.uned.es

\section{Ramón Ramos \\ Instituto Complutense de Sociología para el Estudio de las Transformaciones Sociales Contemporáneas (TRANSOC) \\ Departamento de Sociología I. Facultad de Ciencias Políticas y Sociología. Universidad Complutense de Madrid. España / Spain \\ rr@@cps.ucm.es}

Recibido / Received: 01/04/2016

Aceptado / Accepted: 12/11/2016

\section{RESUMEN}

Este artículo estudia los discursos sobre la confianza de los legos, no expertos, actores sociales comunes en España. Su punto de partida es una investigación sobre las maneras de entender el futuro relativamente próximo (5 010 años) en el contexto de la crisis socioeconómica española. El material para el análisis es aportado por nueve grupos de discusión formados por sujetos muy distintamente afectados por la propia crisis socio-económica y sus riesgos. Aun cuando las referencias al término "confianza" son escasas durante el discurso espontáneo de los actores, muestra la coexistencia de cierta carencia de confianza en las instituciones $y$, a la vez, confianza en la sociedad. Se concluye que, más allá de las circunstancias de la crisis, los actores mantienen una crítica y condicional confianza en la sociedad como forma de enfrentarse a las incertidumbres y riesgos con respecto al futuro.

\section{ABSTRACT}

This article studies the discourses on trust of lay, non-expert, social actors in Spain. It is centred in a research on the way of understanding the next future within the context of the Spanish socialeconomical crisis. The material for the analysis was supplied by nine focus groups formed by participants that were differently affected by the crisis and social-economical risks. While finding out that the term "trust" is rarely used during the spontaneous discourse of the actors, it shows the coexistence of a certain lack of trust in the institutions but trust in society. It is concluded that beyond the circumstances of the crisis, the actors preserve a critical and conditional trust in society in order to face the uncertainties and risks put in their future.

Palabras clave: confianza, crisis, futuro, riesgo, incertidumbre.

Keywords: trust, crisis, future, risk, uncertainty.

\footnotetext{
*Autor para correspondencia / Corresponding author: Javier Callejo. Facultad de Ciencias Políticas y Sociología. Universidad Nacional de Educación a Distancia, Obispo Trejo 4, 28007 Madrid.

Sugerencia de cita / Suggested citation: Callejo, J., Ramos, R. (2017). La cultura de la confianza en tiempos de crisis: análisis de los discursos. Revista Española de Sociología, 26 (2), 185-200
}

(http://dx.doi.org/10.22325/fes/res.2017.12) 


\section{INTRODUCCIÓN}

La confianza ha interesado siempre a la sociología pues constituye un punto clave en la tensión constante entre preservación y aumento de la diversidad —y la libertad — y el mantenimiento del orden social (Misztal, 1996:25). Ciertamente su presencia ha tenido distinta intensidad y sufrido fluctuaciones, de manera que cuando parece arrinconada en un segundo plano (como un concepto vinculado al pasado, a la comunidad tradicional) acaba volviendo y situándose en el centro de los problemas de la teoría y la investigación empírica de la sociología. Sin embargo, cuando se pasa de la reflexión teórica a las percepciones y representaciones de los ciudadanos, la cosa parece más abierta, especialmente en momentos de crisis económica, como en la España actual. Las preguntas que surgen son muchas. He aquí una mínima muestra: ¿Qué sentido tiene la confianza en la sociedad contemporánea? ¿Aparece como tema relevante en situaciones de crisis? ¿La plantean los sujetos? ¿En qué confían y de qué desconfían?

El concepto de confianza funciona en dos niveles (Thuderoz, 2003:19): el de los expertos y el de los legos, es decir, el de los analistas-científicos-sociales y el de los actores sociales. Esto supone ventajas y desventajas: ventajas porque permite utilizar indicadores de confianza directamente derivados de las preguntas integradas en cuestionarios estandarizados a los que responden amplias muestras de población; pero también desventajas tales como su indudable polisemia (Löfstedt, 2005:6), con frecuencia muy cargada moralmente.

Es el nivel lego el que centra la atención de este trabajo. En concreto, pretende aproximarse al uso espontáneo del concepto, evitando la imposición de un concepto experto a una población que tal vez no lo use o lo use de forma muy distinta - como ocurre con frecuencia en las encuestas con cuestionario estandarizado (Bourdieu, 2002). Con ese propósito, se ha construido un contexto o situación discursiva con una doble característica. Por un lado, se ha trabajado con categorías de actores sociales seleccionados por estar ubi- cados en distintas situaciones de riesgo. Por otro lado, se ha situado discursivamente a los sujetos investigados ante el reto de dar cuenta de sus expectativas de futuro (próximo).

\section{CONFIANZA Y MODERNIDAD}

Son muchas las sociologías que han subrayado la importancia analítica y social de la confianza, caracterizándola ya sea como hecho básico de la vida social (Luhmann, 1996:5; 2000), ya como lubricante de las inevitables fricciones de la vida social (Putnam, 2000:135), ya como característica definitoria de las sociedades avanzadas (Stzompka, 1999). No es cuestión aquí de hacer el repertorio de los distintos conceptos construidos, ni dar cuenta cumplidamente de su centralidad en la teoría sociológica. Baste subrayar que la confianza se considera como algo dotado de una sustancia y lógica propias: una acción social con su específica estructura ( $C_{0}$ leman, 2011) o una relación social con su propio código (Luhmann, 1996) 0 un factor imprescindible para la cohesión social (Putnam, 2000) 0 un prerrequisito para la democracia (Mistzal, 1996). En ella se cree captar algo decisivo que revela tanto el funcionamiento como el estado de la sociedad y que incluso permite, según algunos (Offe, 1999:45), trazar el puente que supera el "micro-macro gap" en la teoría social.

En estos momentos, en que el riesgo se ha encumbrado como denominador distintivo de la sociedad como totalidad — sociedad de riesgo en: Beck (1998), Giddens (1990, 1991, 2000), Luhmann (1992), Adam et al. (2000), Ramos (1999), Tulloch y Lupton (2003), Mythen y Walklate (2006), Wilkinson (2006), Renn (2008) o Zinn (2008) — y en que, especialmente en el marco de la crisis económica, la sensación de incertidumbre y vulnerabilidad se extiende al conjunto de las categorías sociales (Bauman 2007; Castel 2009), el concepto de confianza adquiere un relieve particular. Lo alcanza tanto por el estrecho vínculo que guarda con tales conceptos —riesgo, incertidumbre, vulnerabilidad - como por la exigencia de observar cómo se configura en tal contexto. Si es verdad que "we actually are 
creatures who habitually tolerate risks" (Douglas y Wildavsky, 1982:79) y que eso es un rasgo importante de nuestra experiencia social, ¿cómo incide esto sobre la confianza?

Hay, además, otros procesos, como el incremento del individualismo, que ya fueron atendidos en la sociología clásica, que acentúan la relevancia de la confianza (Simmel, 2013; Seligman, 1998; Putnam, 2000). En efecto, en la modernidad, aumenta la interacción entre individuos y la formación de grupos y nuevas comunidades-asociaciones cada vez más diferenciados (Putnam, 2000; Sztompka, 1999; Rothstein, 2005), lo que parece hacer aumentar orgánicamente la necesidad de confianza.

¿Aumenta, en consonancia con estas hipótesis, el grado de confianza general expresado efectivamente por los actores inmediatos? La pregunta es insoslayable, pero conviene posponer su contestación categórica.

En efecto, la confianza requiere la autonomía del sujeto (Luhmann, 1996); a diferencia de lo que ocurre en las sociedades tradicionales, presupone la libertad de los otros, la contingencia de su acción. Pues, en efecto, la confianza deriva de que: "el otro puede hacer cualquier cosa, puede comportarse como quiere" (Seligman, 1998). De este modo confianza e incertidumbre quedan emparejadas.

Pero no se trata tan solo de esto, pues el individualismo está además en conexión con otros procesos. En efecto, un mundo social más individualizado y diferenciado resulta no solo más complejo y opaco, sino también más abierto a novedades insospechadas y a inestabilidades. Todo esto aboca a una constante toma de decisiones 'a ciegas'. En este contexto, la confianza se convierte en un dispositivo fundamental, pues permite abordar con una cierta seguridad y alguna expectativa de éxito las relaciones con lo nuevo (Giddens, 1990) y/o lo contingente (Luhmann, 1996).

Debido a la necesidad funcional de confianza, algunos proponen que las sociedades más eficientes - en términos de desarrollo capitalista- son aquellas que más confían (Putnam, 2000; Fukuyama, 1995). Se trata de una confianza distinta de la propia de las sociedades tradicionales, no tanto proyectada en personas, como en sistemas abstractos. ¿De dónde viene esta confianza en siste- mas abstractos? Las respuestas a este interrogante estratégico son variadas.

La respuesta que algunos proponen (por ej. Fukuyama, 1995) la derivan de la cultura y las costumbres propias de una sociedad, de su específica cultura de la confianza. Desde una perspectiva algo distinta, Inglehart (1999) plantea la confianza como producto del desarrollo de la modernidad y el bienestar económico mediado por la tradición religiosa (católica, protestante, budista, etc.).

Otros conciben la confianza, no ya como soporte o producto natural y a-problemático de la modernidad, sino como un déficit, una carencia que corre el riesgo de convertirse en déficit estructural en la modernidad última. Es algo que se considera a la vez necesario e insuficiente, es decir, un producto que se precisa pero que no se produce (por lo menos no se produce en la medida necesaria), lo que plantea ineludiblemente el problema de la sostenibilidad de ese tipo de sociedad. Resulta así que si la confianza se concebía como elemento central en la modernidad, el déficit de confianza se convierte en el problema crucial de lo que se ha dado en llamar postmodernidad (déficit de confianza en los grandes discursos: Lyotard, 1984) 0, contra ésta, modernidad tardía (Giddens, 1990), modernidad reflexiva (Beck, 1998), etc.

Son varias las dimensiones de esta modernidad tardía apuntadas como fuente del déficit de confianza, además de las que arraigan en procesos señalados antes, pero que ahora cobran mayor intensidad, como, por ejemplo, el aumento del individualismo y el anonimato (desconocimiento de los demás relativamente cercanos en el espacio) o la aceleración del cambio histórico y social o la emergencia de un mundo desbocado (Giddens, 2000; Douglas y Wildavsky, 1982:192). Escenario de fondo que hace hincapié en transformaciones del mundo de vida de los sujetos que lo hacen menos confiable: un mundo en que los sujetos pueden adquirir cualquier rol en cualquier momento, un mundo de roles fluctuantes (Sztompka, 1999) e identidades difusas (Seligman, 1998), donde es difícil tener expectativas sobre la conducta del otro concreto. Algunos hablan de cultura del cinismo (Stivers, 1994; Putnam, 1995), en la que el trabajo de la confianza es, a la vez, mayor y más difícil. Pero mientras que para los proponentes del déficit 
de confianza, ésta es escasa por las dificultades que le opone el mundo social en el que se da, para otros, como Luhmann 0 Giddens, precisamente porque vivimos en un mundo social difícil se incrementa la necesidad de la confianza ${ }^{1}$. En puntos intermedios, como no podía ser de otra manera, hay distintas posiciones, apelando en la mayor parte de las ocasiones a distintos tipos de confianza. Así, por ejemplo, el problema de las dificultades para confiar en los otros cercanos en un mundo complejo se solventa parcialmente confiando en sistemas expertos, abstractos, especializados funcionalmente en un campo de actuación, emocionalmente lejanos y moralmente neutralizados.

Otro de los rasgos que retratan a la modernidad tardía es el escepticismo. Deriva del papel que desempeña la crítica como actitud para configurar y enfrentarse al mundo moderno. El escepticismo se situaría en el núcleo de las sociedades modernas, frente al dogmatismo ritual de las tradicionales. Como propone tan convincentemente Koselleck (2007), el desarrollo de la modernidad no habría hecho sino acentuar el par crítica-crisis proyectándolo sobre las instituciones, de manera que éstas tienen que vivir con una confianza que, lejos de ser absoluta 0 ciega, es asimismo crítica. Ese hecho decisivo se proyecta sobre la confianza en los sistemas expertos. En efecto, si el sistema experto requiere, para su buen funcionamiento, una confianza activa - active trust, Giddens_-, su complementario en sociedades críticas, con ciudadanos críticos, es una critical trust (Taylor-Gooby y Zinn, 2006)².

Muchas concepciones de la confianza, sobre todo aquella que atiende a su transformación allí donde se hace difícil el conocimiento seguro de los otros cercanos, asumen su carácter personalizado.

1 La confianza no supone incremento de información (Giddens, 2000) o de conocimiento (Luhmann, 1996; Earle y Cvetkovich, 1995), sino su déficit. Por eso en un mundo lleno de incertidumbres el mecanismo crucial es la confianza que compensa el desconocimiento 0 la desinformación sufridos.

2 El concepto de confianza crítica tiene especial relevancia en el análisis de la relación de la población con instituciones que regulan el riesgo (Poortinga y Pidgeon, 2003; Pidgeon et al., 2003), como cruce entre alta confianza general y elevado (sano) escepticismo.
Su centro de atención es el modo en que se fragua y mantiene la confianza en y entre las personas. Tomando esta cara del asunto en consideración, la literatura sobre el tema explora la relación entre esta confianza personalizada y la confianza despersonalizada (en instituciones, el otro generalizado, los sistemas expertos, la sociedad en general, etc.) (Mitszal, 1996:21). Así, Fukuyama (1995) plantea la oposición entre sociedades que confían en la familia y otras que confían en la sociedad. En esta tipología, destaca la concepción de la sociedad como entidad colectiva que es, a la vez, agente de confianza (truster) y objeto de confianza (trustee), de manera que la confianza en la sociedad es algo distinto de la confianza en las instituciones.

El concepto de confianza en la sociedad ha generado algunas críticas, como la que denuncia que la confianza de que se habla quede desconectada de la reciprocidad (Barbalet, 2009), lo que implica una crítica extensible a todo tipo de confianza despersonalizada. Más allá de tal crítica, cabe afirmar la existencia de un consenso bastante generalizado en la teoría sociológica sobre el hecho de que, al ubicar la confianza en la modernidad, se extiende la idea de una confianza que va más allá de las personas y su modo de vida. Hipótesis de especial atención para esta investigación, que se propone observar los discursos (más o menos) espontáneos de la confianza social en un contexto discursivo de crisis generalizada - económica, política y probablemente institucional - para retratar las características y contenidos de su uso profano. Tal es el contexto en el que se ha producido la observación empírica, entre los años 2012 y 2013, de la que ahora se presentan los resultados.

\section{OBSERVACIÓN EMPÍRICA DE LA CONFIANZA: ALGUNOS ANTECEDENTES.}

La confianza es un objeto presente en la observación sociológica empírica. Su coincidente necesidad y déficit en las sociedades modernas tensiona investigaciones en las que se pregunta o cuestiona a la sociedad sobre algo que se sospecha está cuestionado por la misma sociedad. La confianza es indagada con frecuencia en las gran- 
des encuestas nacionales, como la General Social Survey, la British Social Attitudes, etc. También en las encuestas internacionales, como las que llevan a cabo el International Social Survey Programme (ISSP) o la European Social Survey. Se pregunta sobre el grado de confianza en el "otro generalizado", en instituciones políticas y en categorías sociales.

Especial relieve, por la importante frecuencia y periodicidad de sus observaciones (que conforman largas series históricas), tiene el cuestionamiento de la confianza en relación con las prácticas económicas: confianza en la situación económica general, confianza de los empresarios, de los consumidores, etc. Son estudios con una elevada estandarización, en muchos casos teniendo el horizonte de la comparación internacional, y una dilatada tradición ${ }^{5}$ que se pueden encontrar en la mayoría de los países desarrollados y cuyo horizonte es la predicción del comportamiento de los agentes económicos.

Más específicas son las encuestas que abordan la confianza en determinados colectivos profesionales 0 la que tienen clientes y consumidores en empresas y determinados productos (Eisenstadt y Roniger, 1984), 0 en los inversores y mediadores en los mercados financieros (Pixley, 2004). Se reconoce así el papel de las emociones en general y de la confianza en particular en las prácticas económicas. La forma de cuestionar sobre la confianza en las instituciones políticas o en los distintos mercados y prácticas económicas se ha extendido en

3 Series temporales desde la A:1.01.02.011 a la B:2.102.05.017 del banco de datos del Centro de Investigaciones Sociológicas, cuando se trata de instituciones; de la F:1.03.01.011 a la F:1.03.01.033 cuando se trata de confianza en la gente: Preguntas A3-A4 y de B1d a B9 de la 7. ${ }^{\text {a }}$ ronda de la European Social Survey. Ocho preguntas (iniciadas con Trst en la base de datos) en la British Social Attitudes de 2014. Serie desde 1972 hasta 2014 sobre la confianza en la gente en la General Social Survey. Las variables relacionadas con la confianza también ocupan un lugar central en la base de datos del ISSP, apareciendo preguntada en sus cuestionarios, siendo, por ejemplo, la Q48 en 2014 y, ahora, la Q11 en 2017.

4 ¿Qué significa para los encuestados a través de esta metodología el término cualquiera cuando se les pregunta si hoy en día se puede confiar en cualquiera?

5 El inicio de estas encuestas es ubicado por Pixley (2004:153) en los años veinte del siglo pasado en Estados Unidos. buena medida al campo de las relaciones entre sociedad y tecnología, como hace Khodyakhov (2007). Así, tomando un ejemplo específico representativo de este tipo de estudio, Am (2011) diferencia entre objetos tecnológicos de confianza y objetos tecnológicos en los que se desconfía. En todos estos estudios, se trata de una confianza cuestionada, en el doble sentido que se ha expuesto antes: se parte de la hipótesis de que la sociedad tiene un déficit de confianza en esos campos (una confianza cuestionada) y se cuestiona (pregunta) por ella.

Un paso adicional lo dan las investigaciones que se adentran en la explicación de la confianza: qué la condiciona 0 , al menos, qué variables están relacionadas con su ausencia/presencia. Cabe incluir aquí estudios como el de Inglehart (1999) sobre la relación entre confianza y bienestar económico, el de Uslaner (2002) sobre confianza y optimismo o el de Brehm y Rahn (1997) sobre confianza y satisfacción en la vida.

Dentro de las relaciones entre sociedad y tecnología, cobran especial relevancia los estudios sobre la conexión entre la confianza y distintas caras del riesgo, especialmente la percepción del riesgo de distintas tecnologías (aun cuando alguno, como Sjóberg (2001), señale que tal relación entre percepción del riesgo de las tecnologías y confianza no se sostiene empíricamente). Otra aproximación busca la relación entre confianza en la tecnología y la percepción de los reguladores del riesgo (Poortinga y Pidgeon, 2003). Así, Kasperson et al. (1992) subraya el carácter tridimensional (percepción de competencia, ausencia de sesgo y compromiso con el proceso e intereses de los demás) de la confianza en los reguladores tecnológicos. Es en el análisis de los resultados de tales estudios donde opera el concepto de confianza crítica ${ }^{6}$, abriéndose la reflexión

6 Concepto que se va asentando en este campo con el apoyo de relevantes estudios cualitativos (Irwin et al. 1996; Poortinga et al. 2003; Walls et al. 2004). En España, destaca el trabajo de Solá et al. (2009), que concluyen como se puede simultáneamente confiar y desconfiar en gestores de un riesgo. Conclusión que apunta a que la distancia entre confianza y desconfianza es escasa y, sobre todo teniendo en cuenta el desarrollo de nuestro trabajo, la posibilidad de convivencia de confianza y desconfianza. 
a las vinculaciones, solapamientos 0 diferencias entre confianza en la tecnología y confianza en los reguladores. La perspectiva tomada aquí difiere, puesto que parte más de la transformación de la confianza, que de un déficit de la misma.

Todos estos estudios van más allá de averiguar cuánta confianza existe, para indagar qué condiciona los grados de confianza. En la medida en que se parte del presupuesto del déficit, se observa a cuánto asciende el mismo y cuál es su fuente principal.

\section{METODOLOGÍA}

Para observar empíricamente las formas legas de concebir la confianza y sobre todo, para saber en qué confían los ciudadanos, en este estudio se ha optado por una aproximación abierta. Así, se ha renunciado a la formulación, más 0 menos estandarizada, que pregunta por la confianza que se tiene en un objeto social determinado: una institución, una categoría social, una profesión o el "otro generalizado". Sin discutir la validez de tales aproximaciones, aquí la observación se centra en el surgimiento de la referencia a la confianza en discursos enfocados hacia dos temáticas que la literatura experta señala como en fuerte conexión con su campo semántico: las expectativas generales de futuro de los sujetos y sus percepciones de riesgo. Como ambos son conceptos fronterizos con el campo semántico de la confianza, cabía esperar -como hipótesis - que las referencias a ésta surgiesen de forma espontánea ${ }^{7}$. Por ello, se optó por una práctica de investigación social discursiva de carácter abierto, como el grupo de discusión, que permitiera abordar el reto comunicativo que supone aproximarse al habla de la gente como si estuviese en sus mundos de vida (Alonso, 1998): cuando la gente habla sobre su futuro y potenciales riesgos isurge la confianza como proble-

7 Todo lo espontánea que se puede en una situación de observación empírica y, por lo tanto, en cierto grado experimental. ma? ¿hay referencias a la desconfianza 0 a cierto déficit de confianza?

La dinámica de los grupos de discusión se impulsó invitando a hablar del futuro próximo; pero, a su vez, evitando el uso de conceptos que podrían tenerse como expertos o extraños a los legos. Así, se presentó el estudio como una investigación sobre cómo se ve la gente en el día de mañana. El estímulo inicial se formuló: ¿cómo se imaginan que será el mundo dentro de diez 0 veinte años? Sin mencionar el término futuro, el primer bloque se focalizó en el futuro personal de los participantes que, tratándose de esta técnica de investigación, comparten ciertos rasgos de sus respectivos mundos de vida. Un segundo bloque se enfocó hacia las preocupaciones o temores en relación con ese futuro, fijando el acento en plantear qué se hace para alcanzarlo 0 evitarlo. La dinámica se hizo con una moderación más directiva en el tercer bloque, en el que se expusieron específicas fuentes de riesgo.

Aun cuando desde la concepción abierta de la dinámica del grupo de discusión se tenía como principio táctico el entrar a hablar de la confianza en cuanto surgiese el término, se reservó un cuarto bloque para preguntar por él directamente, si no surgiera con anterioridad. Después de haber establecido temas de discusión como futuro, riesgos, expectativas, posibilidades de los sujetos u otras similares, la ausencia de referencia al concepto exigiría también una interpretación. Pero ello no eximía, claro está, de preguntar por él de manera directa a los participantes.

En cuanto al diseño de los perfiles de los participantes, se optó por sectores medios de la sociedad, estructurándose sobre la mayor/menor vulnerabilidad, tanto de carácter general (crisis económica), como de carácter específico (algún tipo de riesgo: tecnológico, socioeconómico o urbano-industrial). Teniendo en cuenta que la vulnerabilidad de carácter general tomó como criterio principal la relación con el mercado laboral, se diseñaron los siguientes nueve grupos: 
Tabla 1. Diseño de los grupos de discusión

\begin{tabular}{lll}
\hline PERFIL & EDAD & LUGAR \\
\hline Hombres. Funcionarios capital provincia sin riesgos tecnológicos. & $50-60$ & Valladolid \\
\hline Mujeres. Técnicos y profesionales, en empresas medias. & $35-45$ & Toledo \\
\hline Hombres y mujeres. Estudiantes universitarios, clase media-alta. & $20-25$ & Valladolid \\
\hline Mujeres. Amas de casa, hábitat macrourbano, clase media, con hijos menores. & $35-45$ & Madrid \\
\hline $\begin{array}{l}\text { Trabajadoras (dependientas, peluqueras, etc.), hábitat macrourbano, clases populares, } \\
\text { con hijos menores. }\end{array}$ & $30-40$ & Madrid \\
\hline Hombres. Autónomos (sector oficios: fontanero, taxista, dueño pequeño bar, etc.) & $40-50$ & Madrid \\
$\begin{array}{l}\text { entorno urbano. } \\
\text { Hombres y mujeres. Empleados contrato fijo (empresa grande o media). }\end{array}$ & $30-40$ & Tarragona \\
\hline Proximidad riesgo tecnoindustrial. & $45-55$ & Madrid \\
\hline Varones. Trabajadores sector industrial-químico. & $20-30$ & Madrid \\
\hline Hombres y mujeres. Jóvenes empleados contrato temporal, sin hijos. & & \\
\hline
\end{tabular}

Aunque se han encontrado relevantes diferencias entre los distintos grupos, este trabajo se centra en las convergencias, en lo que tienen más en común, en lo compartido ${ }^{8}$. De aquí que puedan

8 Lo compartido, que no deja de ser el resultado de un proceso de abstracción, es uno de los criterios que, por la vía de la saturación (Callejo, 1998) conduce a la norma discursiva, a lo que Hejmslev (1971) denomina norma, oponiéndolo al uso. En la investigación sociológica cualitativa, se puede tomar como objeto la reconstrucción de la norma, como aquí se ha intentado, o la configuración de los distintos elementos del campo o sistema discursivo, entendidos como puntos de fuerza y en potencial relación de conflicto 0 acuerdo. Las limitaciones de espacio a las que está sujeto un artículo de estas características, nos ha llevado a centrarnos en la primera perspectiva, dejando la segunda para próximos trabajos. Por otro lado y para evitar atajos analíticos (Antaki et al., 2003), creemos que el objetivo de la reconstrucción de la norma precede al de la configuración de diferencias. Hay que resaltar la observación de una doble convergencia: dentro de cada grupo y entre los grupos, tomando en todo caso el recorrido integral de los discursos y poniendo el foco en su emergencia interaccional. Por último, señalar que los matices observados con respecto al discurso dominante o legítimo no pueden considerarse aquí reveladores de oposiciones conflictivas, comprendiéndose mutuamente los participantes en los grupos de discusión que podrían ser situados en el polo de la desconfianza y en el polo de quienes mayor grado de confianza general han expresado. Ambos polos reconocen fundamentos para la confianza — situando como sujeto a la sociedad o sus movimientos sociales - y la desconfianza. entenderse sus conclusiones como un retrato de la cultura de la confianza en un contexto específico, el de la crisis económica.

\section{LA AUSENCIA EN EL DISCURSO ESPONTÁNEO}

La investigación ha constatado la ausencia de referencias espontáneas a la confianza. Un hecho que nos dice que el habla de los sujetos no echa mano del par confianza/desconfianza para abordar el futuro, sus expectativas, los riesgos percibidos 0 las posibles amenazas. Si nos quedáramos ahí, podría derivarse la conclusión de que no hay problemas de confianza 0 , al menos, que los ciudadanos no la utilizan para estructurar un mundo que se ha dibujado con trazos de riesgos y peligros, en una situación que es, como reconocen los propios grupos de discusión desde el principio, de crisis económica. En principio, parece que la gente puede vivir sin plantearse la cuestión de la confianza — 0 la desconfianza - al menos en relación con las instituciones.

Cuando, ya en el bloque directivo de la dinámica, se pregunta por la confianza, nos encontramos con el carácter reactivo de la respuesta, que varía escasamente en el conjunto de los grupos entre las siguientes manifestaciones: "no se puede confiar en nada" o "solo se puede confiar en uno mismo". 
Hay, pues, un marcado contraste entre la ausencia de la confianza en el discurso espontáneo y la intensa reacción en el bloque de la dinámica más dirigida. Es evidente que ese contraste busca interpretación, que hemos de alcanzar a partir de los indicios dejados en el propio discurso.

Una primera interpretación de la ausencia pasa por la insuperable tensión entre el habla de los sujetos en su mundo de vida y ese habla en una situación experimental (grupo de discusión). Desde aquí, cabe apuntar la posible dificultad de los sujetos para ponerse en situación de víctimas 0 en supuestos de un futuro perjuicio o daño. Es cierto que algunas situaciones les son más cercanas —el desempleo, el desahucio- pero, en general, hay una resistencia a verse como víctimas. Si no se pueden poner en tal situación, no pueden plantearse qué harían, a qué o quién acudiría, lo que sería uno de los caminos semánticos para adentrarse en la confianza.

Si la primera interpretación se establece en clave metodológica, la segunda entra en los contenidos materiales de lo dicho. Para empezar, se revela inapropiada la oposición sin más de la confianza y la desconfianza. Tras la primera reacción, que sitúa la confianza fuera del mundo de vida de los sujetos, las siguientes intervenciones tienden al matiz, a esbozar una dialéctica entre una confianza plena, que se reconoce como un ideal ausente de la realidad, y una confianza relativa.

- La confianza ...

- Pero nunca va a haber esa confianza que queremos.... Porque no....

- - pero si hay alguna duda... una buena formación y unos buenos cimientos, puede soportar cualquier envite....

- Claro es que tú en principio lo planteas como que si... Yo hablo mucho con ellos, ... (Funcionarios Valladolid)

- Yo pienso que en el momento que tu hijo, por ejemplo, por ponerte un caso, el Madrid Arena que está ahora tan reciente. Tú dejas ir a tu hijo, tú tienes confianza en tu hijo, eso es lo primero. Tú dejas ir a tu hijo a una fiesta 0 a una... discoteca... tú no sabes lo que está haciendo tu hijo. Por mucha confianza que tengas con tu hijo, por mucha educación que le hayas inculcado a tu hijo. Tú no sabes, no tienes una cámara para ver lo que está haciendo tu hijo dentro de un local. Juegas con que tú tienes esa confianza, tiene tu hijo esos valores contigo también, pero tú no sabes lo que está haciendo exactamente tu hijo, tú tienes que tener confianza en tu hijo. Entonces en el momento que el sale, o ella sale.... No lo puedes controlar.... (Técnicas y profesionales Toledo)

Hay una confianza realista, en cuanto necesaria, que utiliza el pronombre que indica el sujeto de derechos y obligaciones —el "tú" — que está llena de incertidumbre ("no lo puedes controlar"). Matización de la primera reacción a la (des)confianza: no hay una confianza plena, sino una confianza relativa y concreta. Hay una lógica digital de la necesidad de la confianza (se necesita — " tú tienes que tener confianza" - 0 no se necesita la confianza) y una lógica analógica, en cuanto incierta, de las consecuencias o resultados de la confianza: no lo puedes controlar, pues esos resultados cabe esperarlos en mayor 0 menor grado. A la vez, las respuestas sobre la confianza dan la espalda a la cuantificación; ni siquiera se responde en clave de mucha, poca 0 bastante confianza ${ }^{9}$. Es una confianza incierta (en lo que pueden considerarse sus resultados: el comportamiento final del objeto de confianza), pero no en cuanto a su posesión, pues se tiene 0 no se tiene confianza. $Y$ si se necesita, se tiene (o no se tiene).

Ahora bien, si partimos de la oposición entre confianza en personas y confianza en instituciones (Offe, 1999), como concreción de la confianza despersonalizada, constatamos que la primera reacción está parcialmente personalizada. Es una (des) confianza en "nadie", lo que conlleva un rechazo personalizado: no se confía en los que se conoce,

- La verdad es que yo no confío ya en nadie

- Confiar ... en nadie (Jóvenes precarios Madrid)

9 Solo, más tarde y cuando se hable de la confianza en la sociedad, aparece el término de confiar un poco. 
Incluso se rectifica la despersonalización-institucionalización que contiene la pregunta del moderador de las reuniones, para subrayar la personalización.

- Moderador: Bien... un poco habéis planteado como veis generalmente la situación del futuro... ¿Y en qué se confía?

- En nada

- Yo no confío en nadie

- Ni yo (Técnicas y profesionales Toledo)

Pero se trata de una personalización matizada que desemboca señalando, no tanto falta de confianza, como una fuente personalizada de desconfianza: los políticos. Aún no sabemos en quién o qué se confía, ni en quién 0 qué se desconfía 0 no se confía, pero los políticos aparecen como fuente de desconfianza personalizada, junto a algunas instituciones, no ya directamente políticas. Es lo que aparece en el siguiente fragmento discursivo, en el que hay que señalar algo importante, como es el cambio en el sujeto que actúa, que pasa del yo al nosotros:

- Día a día

- En nadie

- Políticamente, en nadie

- Yo no confío en nadie

- Política, social y ...

- Luego socialmente... lo mismo... entidades bancarias y todo este tipo de cosas, luego te las hacen por todos los lados. Lo que decías anteriormente, que si el "hombre de negro", que si no se qué... que si mobing, que si la madre que lo parió, que si la Unión Europea, que si tal.... Pues la desconfianza creo que ha llegado al nivel de masa, dentro de la población lo que es por lo menos de aquí, de nosotros.

- Es que hemos llegado al punto este...

- ... por la calle con cualquiera que hables...

- Los bancos están... a las personas para sacar el dinero. Es que no es decir, bueno es que el banco te va a apoyar $0 . .$. ; es que van a engañar (Autónomos Madrid)

La figura de "Ios políticos" concentra la desconfianza en la medida que personaliza la respon- sabilidad. Se les hace responsables de casi todo lo que acontece, desde los altos salarios de los futbolistas a la propia falta de confianza en la justicia. La desconfianza funcional en los políticos, tanto en su competencia como en su moralidad, se dice desde el nosotros.

- De políticos... yo es que realmente no voto... porque me da igual... todos son muy buenos hasta que les das el cargo. Una vez que les das el cargo... porque (Jóvenes precarios Madrid)

Se establece una oposición entre un "ellos" y un nosotros. Entre estos dos polos, que pueden tomarse como personalizaciones de la oposición entre desconfianza y confianza, se instalan diversas categorías sociales. Cerca del segundo polo, se sitúa a los padres, y, especialmente entre las mujeres, a las madres. Sobre la propia pareja se manifiesta una confianza relativa, incierta, volátil: se confía mientras dure, pero no hay inversión incondicional de confianza como en padres y madres. Los hermanos ocupan una posición marginal; también, sorprendentemente, los abuelos (empleados Tarragona), tal vez un caso de falta de confianza funcional. En todo caso, contrasta la débil confianza en todas estas categorías familiares - pareja, hermanos, abuelos - cuando la pregunta se hace abierta, con las muestras de confianza en la familia que recogen las encuestas con cuestionario estandarizado ${ }^{10}$.

En relación con los amigos, se muestra de forma manifiesta lo que podría considerarse el carácter ambiguo de la confianza en el mundo de vida de los sujetos. A diferencia de lo que ocurre con la familia, la figura del amigo concreta como ninguna el concepto de confianza que tiene la sociedad, la confianza que se da. Frente a la confianza en la familia o los miembros de la familia, que es la confianza en la que se está como un hecho natural o que hay que dar necesariamente, por imposición moral, la confianza en el amigo es una confianza elegida, decidida; ser amigo consiste en confiar; confianza y amistad son dos caras de lo mismo.

10 Por ejemplo, en el Latinobarómetro de 2013, el 89,6\% de las personas entrevistadas seleccionaban la opción mucha confianza en su familia. 
De aquí que cuando surge el tema de la confianza en los amigos, tienda a resolverse con la descripción normativa de lo que debe de ser un amigo:

- Son amigos míos de hace... pero de niños... hace 40 años

- ... uno, uno y pico

- Uno coma cero cinco.

- No quiere decir eso que... como le ha pasado lo mismo ya tiene cierta experiencia en muchas cosas, te han dado por culo porque te han dado, entonces guardas las espaldas...

- Amigos, amigos realmente no se tienen muchos.... Tienes conocidos, pero amigos, amigos...

- No, yo digo amigo a alguien que te diga: Oye mira, aquí tienes mi casa y quédate un tiempo... por ejemplo...

- Eso es un amigo

- ... o lo que necesites... Ilama a mi puerta que siempre va a estar abierta... adelante. Ahora, un amigo no es el que está tomando una caña contigo, te tomas dos raciones, ji, ji, ja, ja... (Autónomos Madrid)

Se asume que un amigo es un bien escaso: son pocos los amigos, pocos en los que se puede confiar. Y para argumentarlo se recurre a la oposición entre lo ideal y la realidad _- "realmente no se tienen muchos" —; una realidad cualificada además por incertidumbres reconocidas que se expresan en un tópico lapidario: un amigo no sabes que lo es hasta que no lo necesitas.

El mundo del trabajo es el que aparece más afectado por la crisis. En él opera el par confianza/desconfianza, que aparece en las descripciones que se hacen sobre el centro de trabajo y los compañeros. En los centros, la competencia entre compañeros en tiempos de crisis produce una profunda desconfianza en las relaciones con el otro (un otro que me puede quitar el trabajo 0 promocionarse a mi costa). La desconfianza en relación a los compañeros se hace especialmente expresiva: no se pueden contar las cosas a nadie porque no se sabe qué hará; hay que ocultar los propios propósitos e intereses. En definitiva, en la empresa no hay amigos y no se puede contar con nadie.

- Yo, en el mío sí, es una tienda de ropa y vamos a comisión... lógicamente si entra una señora, estamos las 4 aquí dependientes, la que la pille la ha pillado.... Esto es como todo.... Y si te vas a bajarle algo, cuando vuelves ya está la otra: №, mire llévese esto... Porque ya me la ha quitado, con lo cual... siempre estamos juntas porque estamos ahí trabajando, pero cada una va a lo suyo. Y si yo puedo llevarme más que tú... me lo voy a llevar.... Entonces estamos ahí (Trabajadoras con hijos Madrid)

- ... Yo por ejemplo no tengo mala suerte, pero es que mi amiga.... Dos trabajos seguidos que ha tenido, de por charlar con las compañeras y tal... y se ha llevado puñaladas traperas, pero por todos los lados.... Es lo que yo digo; № te hagas amiga de nadie, tú vete a trabajar y no hables con nadie... ¿sabes? La gente va como, con la malicia ya... de a ver si me vas a quitar el trabajo... no van con buena intención.... Se desconfía más... (Jóvenes precarios Madrid)

\section{La confianza en la sociedad}

La situación parece inicialmente dramática. № se confía en las instituciones ("en nada"), ni en la personalización de las instituciones ("en nadie"); y se confía poco en las personas cercanas. Pero esto no es todo.

$Y$ en efecto, tras la primera reacción que desconfía absolutamente y con intensidad emocional de las instituciones, emerge una reflexión orientada a mostrar fuentes de confianza pública. Reflexión que, en la dinámica grupal, aparece inicialmente con titubeos y que, al ser acogida por el resto de los participantes, toma fuerza. Se pasa así del no confiar en nadie, a establecer diferencias, y del no confiar en nada, a vislumbrar la emergencia de un sujeto colectivo, a la vez, general y difuso, que va ganando concreción según 
se desarrollan las reuniones. Empieza a ser nombrado como la gente:

- Moderador: Pero más allá de eso... he preguntado ¿en quién se confía?... ¿No se confía en nadie...? ¿vais por la calle y vais sin confiar en nadie, ni en nada?

- Yo confío a lo mejor cada vez más en la gente, la gente se está volviendo más social, intenta ayudar a la gente.... Ya en el tema de desahucios y tal ves cómo hay gente que se une para luchar contra ello... a lo mejor no es su casa y no conocen a la persona pero, como que ya se van uniendo. Entonces yo creo que cada vez vas confiando un poco más en la gente, a lo mejor más que antes. Porque antes no pensabas que si a ti te iban a desahuciar iba a ir alguien a ayudarte que no te conociera... sabías que iban a ir tu familia, tus amigos, pero gente así de la calle... no. (Jóvenes precarios Madrid)

Resaltamos la utilización expresa del pronombre de primera persona, el yo, como indicador de la dominancia de la función expresiva del lenguaje (Jakobson, 1975). Pues no solo se manifiesta una opinión, más o menos distante, sino un compromiso con lo dicho cuando se utiliza el "yo confío".

Se trata de una confianza en la gente-sociedad y, más concretamente, en la sociedad que se moviliza, en la sociedad en movimiento por objetivos que van más allá de los intereses individuales inmediatos — no es su casa, no conocen a la persona-, más allá de la desconfianza en las instituciones políticas y en los políticos en particular; una confianza que se brinda a la sociedad como actor colectivo concebido como encarnación de una moralidad positiva, tal como muestra este fragmento:

[...] Un poco saber, en qué sociedad vives. ¿Qué vas a ganar tú con quedarte todo? Pues que al final nadie va a querer estar a tu lado. Un poco compartir. No volverse egoísta. (Jóvenes universitarios Valladolid).

La sociedad aparece como altruismo, puesta en común, compañía del otro — frente al individualis- mo posesivo que acapara objetos y lleva al aislamiento. Sin ella no se puede vivir. Y es por lo que hay que brindarle confianza. Lo demandan la esperanza y el deseo, pero lo justifica también el modo en que se muestra y hace patente cuando "la gen$t e$ " actúa o cuando hay movimientos sociales, como los movimientos anti-desahucios o el 15-M. Ambos son significantes de una sociedad, entre latente y manifiesta, en la que se puede y debe confiar.

Por eso las manifestaciones de protesta y ciertos movimientos sociales se convierten en muestra de que "la sociedad está ahí":

- Si sacas lo positivo de la situación, pues te das cuenta de quién verdaderamente está de verdad y quién no. Entonces habrá que buscar el lado positivo... que te das cuenta de muchas[cosas] que antes... no te dabas cuenta. (Trabajadoras con hijos Madrid)

- Yo estoy bien, pero al de al lado mío le falta de esto, voy a intentar darle un empujón. El no ser egoísta. (Empleados Tarragona)

- Hombre, una pizca de esperanza se tiene. De que quien está ahí, que contribuya, que no es que te saque, también hay que poner de tu parte. Pero, si no, estaríamos en la calle, diciendo: "quita de ahí que no me fío de lo que vas a hacer". Digo yo, que algo hay ahí, un mínimo. Porque es que si no, ¿qué te queda? (Funcionarios Valladolid)

En definitiva, la confianza en la sociedad es el último resorte. Si no se confiara se desplegaría una especie de lucha hobbesiana de todos contra todos y solo cabría la confianza en "uno mismo" y en nadie y nada. Además, es la confianza en la sociedad la que permite recuperar la confianza en las instituciones.

- ¿confiar...? ¿en qué confiamos a parte de uno mismo?

- En la suerte

- No ya no es en la suerte

- En lo que podamos, en los recursos que tengamos y en sacar adelante las cosas sin tomar decisiones dentro de nuestras posibilidades. 
- Pero esto no puedes convertirlo en algo individual, en una lucha individual, así no vamos a ningún lado. No te digo la palabra lucha de... no sé... me gustaría creer en las instituciones, y no creo. Es que como individualmente no puede hacerse nada. Por eso tenemos un Parlamento y tenemos unas instituciones... (Técnicas y profesionales Toledo)

Hay que subrayar la lógica de la reciprocidad que se establece, asimilable a la reciprocidad generalizada que plantea Putnam como núcleo de una cultura basada en la confianza. La confianza en la sociedad como confianza de la sociedad: solo si se confía en la sociedad, la sociedad puede confiar en mí. Vista así, la sociedad no es una especie de "alter ego", un "otro", sino algo muy diferente: un "nosotros".

- Cuando has empezado a hablar de este tema, fíjate en el ánimo de la sala. Nos hemos venido todos abajo.

- La culpa es mía.

- No, es de la situación. Cuesta ver la solución. 0 a mí personalmente.

- Pero tenemos que tirar del carro nosotros, que somos unos privilegiados.

- Sí, claro.

- Hay que poner confianza en los que tienen... por ejemplo, nosotros somos la siguiente generación... vosotros habéis tirado, nosotros tenemos que tirar más, estrujar más.

- A ver, yo, en lo que confío siempre... hay un porcentaje muy pequeño de la sociedad, muy pequeño. Pero siempre hay un porcentaje de la sociedad. Que siempre está dispuesto a cambiarla, y acaba cambiándola. Yo siempre confío en eso. (Empleados Tarragona)

En definitiva, el resultado que alcanzamos es que la confianza que suponen los hablantes de los grupos de discusión es compleja, cambiante, y ciertamente, como toda confianza (Luhmann, 1996), incierta, incluso crítica y total 0 parcialmente despersonalizada. Pero lo importante es que actúa desplegándose y particularizándose en tres instancias diferenciadas:

a) Las instituciones, especialmente las instituciones políticas y sus agentes específicos, los políticos.

b) El otro individualizado o los individuos, desde los más cercanos y con cara reconocible, hasta los más lejanos y solo tipificables. Son los otros de las intervenciones de los grupos o el "cualquiera" de los cuestionarios sobre confianza.

c) El nosotros colectivo e idealizado que se muestra en la acción colectiva, ya sea efectiva 0 potencial. Es el depósito de los valores positivos (solidaridad, igualdad, justicia, etc.) que, en situaciones de normalidad, se resiste a actuar. Aunque idealizada (y por ello más potencial que real), es concebida como una realidad agente, que en el lenguaje de los grupos te dice que está ahí.

La desconfianza se proyecta especialmente sobre la primera instancia, al menos los pronunciamientos más explícitos y radicales de falta de confianza del tipo no confío en nada. A la segunda parece hacérsele cómplice 0 víctima de la primera, y, en esta medida, se opone a la confianza en la sociedad. Es la instancia del "otro individualizado" que tiende al individualismo-egoísmo y entorpece la acción colectiva del "nosotros". La "sociedad" desconfía de una sociedad reducida a puro entramado de acciones individuales egoístas. El viejo tópico del dulce comercio como fuente de sosiego, confianza y calculabilidad parece diluido. El "otro" que domina la segunda instancia no es el "otro generalizado" que Giddens (1984:54; 1991) representa como una figura maternal, sino que adquiere la forma del hombre-lobo hobbesiano. De ahí sus conexiones no sólo con cierta cultura económica (vicios privados/públicas virtudes) en la que no se cree, sino también con una cierta cultura política que edifica la confianza institucional sobre el cálculo de intereses de individuos egoístas.

La tercera instancia es la que resulta propiamente el espacio en el que se deposita, genera y reproduce la confianza. En consecuencia, la sociedad en la que se confía consigue un retrato nítido. № 
es la sociedad civil organizada, ya sea en partidos políticos, asociaciones, organizaciones, etc., tal como los tiende a entender la literatura politológica. Tampoco es una sociedad de individuos que interactúan e intercambian en mercados de distinto tipo. Es, por el contrario, el "nosotros", la sociedad cívica, solidaria, casi comunitaria en su representación imaginaria. Una sociedad que se mueve por valores y con conciencia de unidad, y en la que uno queda integrado y de la que es parte.

\section{CONCLUSIONES}

Hemos destacado que los grupos de discusión no han hecho referencia espontánea a la confianza. Y eso a pesar de lo que suelen mostrar las encuestas con cuestionario estandarizado 0 de lo esperable en una situación reconocida como crítica e incierta.

Para dar cuenta de esa llamativa ausencia, se podría conjeturar que, lejos de ser algo actual en la sociedad española, el problema de la confianza/ desconfiaza lo genera más bien el sistema experto de politólogos y sociólogos, que lo impone socialmente al preguntarlo en las encuestas.

En cumplimiento del diseño de la investigación, se preguntó directamente por la confianza a quienes espontáneamente no la mentaban. La primera reacción muestra falta de confianza en las instituciones y, de forma personalizada, en los políticos. Se trata además de una reacción inicial muy cargada emocionalmente. No se habla de la confianza en las categorías sociales próximas y más o menos personalizadas — familiares, amigos_-, sino en categorías despersonalizadas (instituciones, políticos, etc.).

Resulta pues que, una vez requeridos, a los participantes no les resulta difícil abordar discursivamente la confianza despersonalizada. Es lo que tienden a recoger las encuestas con cuestionario estandarizado.

La confianza despersonalizada parece más dominada por la lógica de la reflexión, que por la de la emoción. Esto puede explicar que no aparezca en el discurso espontáneo y requiera una apelación expresa que lleva a una reflexión que acaba hablando de ella. En cualquier caso, ese discurso sobre la confianza despersonalizada en general y aún más, sobre la confianza en la sociedad o el sistema social en particular, no aflora fácilmente. $\mathrm{Ni}$ siquiera en un contexto discursivo que nuestro diseño metodológico consideraba apropiado. Parece cumplirse la afirmación de Luhmann: "la confianza sistémica difícilmente se hace un asunto abierto a la discusión pública y, asimismo, el hecho de que sea algo latente, ayuda a mantener su integridad" (Luhmann, 1996:99).

Lo que aparece inicialmente como una abrupta declaración general de falta de confianza se torna en el discurso reflexivo en confianza en la sociedad, en una alteridad general e igualitaria, pues se tiende a confiar en los que son como yo y a los que se debe asimismo declarar que pueden confiar en uno. La confianza se torna así en básica y en recíproca. Es una confianza que se considera necesaria para seguir siendo sociedad y de la que puede renacer la confianza en las instituciones.

Interpretando los discursos de los grupos, esa confianza tiene las características de una confianza que hemos denominado crítica. Se asume como necesaria, pero en un marco de incertidumbre y relatividad. Incluso se asume que es una confianza con riesgos, pues toda confianza supone asumir un riesgo (Sabel, 1993; Luhmann, 1996; Das y BingSheng, 2004; Coleman 2011).

Parece que se confía, pues, porque hay que confiar, pero atendiendo también a los mensajes de la propia sociedad. Así, se toman como mensajes determinadas acciones de algunos movimientos sociales (15-M; resistencia a desahucios). Son significantes del retorno de la sociedad: como si volviese a ser digna de confianza, tras haber estado ausente durante tiempo. En todo caso, es una confianza relativa pues se da a una sociedad de la que, hasta hace poco, se desconfiaba.

Esta articulación entre confianza y desconfianza no parece nueva. Es más, puede interpretarse que encarna lo que distintos autores (Denters et al., 2007:66; Klingemann, 1999) han caracterizado como cultura política contemporánea: un fuerte apoyo a los ideales democráticos, que en nuestro estudio se condensa en la confianza en la sociedad como su encarnación, y, por otro lado, actitudes 
críticas hacia las prácticas políticas concretas. Lo que aquí se ha configurado como confianza en la sociedad contendría esa doble cara.

Por lo tanto, se podría decir que no es que la crisis haya generado falta de confianza en las instituciones. Ésta ya era una confianza crítica. Puede decirse, en todo caso, que la crisis ha acentuado la crítica de las instituciones 0 que ha acelerado el proceso de confianza crítica. Pero ha sido esa misma crisis y la aceleración de la debilidad de la confianza en las instituciones la que ha promovido la mirada en busca de confianza a la sociedad y el orden social, buscando signos en ella para poder proyectarla. Cuando los signos aparecen, aflora una confianza general en el sistema, en el marco de desconfianzas concretas, incluso cuando éstas sean masivas (como en uno de los grupos). Pero son desconfianzas masivas sobre un fondo de confianza en la sociedad. Volvemos aquí a recurrir a Luhmann (1996:132), que apunta la retroalimentación entre un inevitable comportamiento desconfiado de los miembros de las sociedades modernas en múltiples ámbitos, y la confianza sistémica.

Por ello, reiteramos la propuesta: parece inapropiado describir nuestra sociedad - la española en situación de crisis- como inmersa en un déficit estructural de confianza, como si fuera una sociedad sin confianza en sí misma. No parece estar instalada en lo que Sztompka (1999) denomina una cultura de la desconfianza, en la que estaría atrapada, según los resultados de las encuestas con cuestionario estandarizado, la mayoría de la gente en las actuales sociedades avanzadas. Junto a la manifestación de actitudes críticas hacia las instituciones, especialmente hacia las que en mayor medida traducen el sistema político democrático, se mantiene una cultura de confianza básica, que puede asumirse como propia de la modernidad porque, como apuntan nuestros grupos, es necesaria para sostener (o restaurar) el modelo de vida que se quiere y espera preservar 0 conseguir.

\section{AGRADECIMIENTOS Y FINANCIACIÓN}

El presente artículo es uno de los resultados de la investigación "Riesgo, incertidumbre y vul- nerabilidad en España", cuyo IP es Ramón Ramos, habiendo estado financiada por el Plan Nacional de Investigación con la referencia CSO-2010-20135.

\section{REFERENCIAS BIBLIOGRÁFICAS}

Adam, B.; Beck, U., Van Loon, J. (2000). The risk society and beyond, Critical issues for social theory. London: Sage.

Alonso, L. E. (1998). La mirada cualitativa. Madrid: Fundamentos.

Am, T. G. (2011). Trust in Nanotechnology? On Trust as Analytical Tool in Social Research on Emerging Technologies, Nanoethics, 5, 15-28.

Antaki, C.; Billig, M.; Edwards, D.; Potter, J. (2003). El análisis del discurso significa analizar: Crítica de seis atajos analíticos, Athenea Digital, 3, 14-35.

Barbalet, J. (2009). A characterization of trust, and its consequences. Theory and Society, 38, 367-382.

Bauman, Z. (2007). Miedo líquido. Barcelona: Paidós.

Beck, U. (1998). La sociedad del riesgo. Barcelona: Paidós.

Bourdieu, P. (2002). Questions de Sociologie. Paris: Minuit.

Brhem, J. y Rahn, W. (1997). Individual-level evidence for the causes and consequences of social capital. American Journal of Political Science, 41(3), 999-1024.

Callejo, J. (1998). Los límites de la formalización de las prácticas cualitativas de investigación social: la saturación, Sociológica, 3, 93-120.

Castel, R. (2009). La montée des incertitudes. Paris: Seuil.

Coleman, J. S. (2011). Fundamentos de teoría social. Madrid: Centro de Investigaciones Sociológicas.

Das, T. K., Bing-Sheng, T. (2004). The risk-based view of trust: a conceptual framework. Journal of Business and Psychology, Vol. 19 (1). Fall, (2004), 85-116.

Denters, B., Gabriel, 0., Torcal, M. (2007). Political confidence in representative democracies. En Jan W. van Deth, José Ramón Montero y Anders 
Westholm (eds.). Citizenship and Involvement in European Democracies. A Comparative Analysis (pp. 66-87). Oxford: Routledge.

Douglas, M., Wildavsky, A. (1982). Risk and Culture. Berkeley. Los Angeles, London: University of California Press.

Earle, T. C., Cvetkovich, G. T. (1995). Social Trust. Towards a Cosmopolitan Society. London: Praeger.

Einsenstadt, S. N., Roniger, L. (1984). Patrons, Clients and Friends. Interpersonal Relations and the Structure of Trust in Society. North Carolina: Wake Forest University.

Fukuyama, F. (1995). Trust. The Social Virtues and the Creation of Prosperity. New York: The Free Press.

Giddens, A. (1984). The Constitution of Society. Oxford: Polity Press.

Giddens, A. (1990). The Consequences of Modernity. Stanford, CA: Stanford University Press.

Giddens, A. (1991). Modernity and Self-Identity. Stanford, CA: Stanford University Press.

Giddens, A. (2000). Un mundo desbocado. Madrid: Taurus.

Hardin, R.(1996). 'Trustworthiness', Ethics107,26-42. Hardin, R. (1988). 'Trust in Government', in M. Levi and V. Braithwaite (eds.). Trust and Governance (pp. 9-27). New York: Russell Sage Foundation.

Hejmslev, L. (1971). Prolegómenos de una teoría del lenguaje, Madrid: Gredos.

Inglehart, R. (1999). Trust, well-being and democracy, en M. E. Warren (ed.). Democracy and Trust (pp. 88-120). Cambridge: Cambridge University Press.

Irwin, A., Dale, A. y Smith, D. (1996). Science and Hell's Kitchen: The local understanding of hazard issues. En A. Irwin y B. Wynne (eds.). Misunderstanding Science? The Public Reconstruction of Science and Technology. Cambridge: Cambridge University Press.

Jakobson, R. (1975). Ensayos de lingüística general. Barcelona: Seix Barral.

Kasperson, R., Golding, E. D. y Tuler, S. (1992). Social Distrust as a Factor in Siting Hazardous Facilities and Communicating Risks. Journal of Social Issues, 48 (4), 161-187.

Khodyakhov, D. (2007). Trust as a process: A threedimensional approach. Sociology, 41 (1), 115-132.
Klingemann, H.-D. (1999). 'Mapping Political Support in the 1990s: A Global Analysis', in P. Norris (ed.). Critical Citizens: Global Support for Democratic Governance. New York: Oxford University Press. pp. 31-56.

Koselleck, R. (2007). Crítica y crisis: un estudio sobre la patogénesis del mundo burgués. Madrid: Trotta.

Löfstedt, R. (2005). Risk Magement in Post-Trust Societies. London: Earthscan.

Luhmann, N. (1992). Sociología del riesgo. Guadalajara (Méx.): Universidad Iberoamericana y Universidad de Guadalajara.

Luhmann, N. (1996). Confianza. Barcelona: Anthropos.

Luhmann, N. (2000). La realidad de los medios de masas. Barcelona: Anthropos.

Lyotard, J.-F. (1987). La condición postmoderna. Madrid: Cátedra.

Misztal, B. (1996). Trust in Modern Societies. Cambridge: Polity Press.

Mythen, G., Walklate, S. (eds.) (2006). Beyond the risk society. Maidenhead UK: Open University Press.

Offe, C. (1999). How Can We Trust Our Fellow Citizens? En M. Warren (ed.). Democracy and Trust (pp. 42-87). Cambridge: Cambridge University Press.

Pidgeon, N. F., Kasperson, F. R. E. y Slovic, P. (2003). The Social Amplification of Risk, Cambridge: Cambridge University Press.

Pixley, J. (2004). Emotions in Finance. Cambridge: Cambridge University Press.

Poortinga, W., Pidgeon, N. F. (2003). Exploring the Dimensionality of Trust in Risk Regulation, Risk Analysis, 23 (5). 961-972.

Putnam, R. (1995). Tuning In, Tuning Out: The Strange Disappearance of Social Capital in America. Political Science and Politics, 28 (4), 664-683.

Putnam, R. (2000). Bowling Alone. The Collapse and Revival of American Community. New York: Simon and Schuster.

Ramos Torre, R. (1999). Prometeo y las flores del mal: el problema del riesgo en la sociología contemporánea. En R. Ramos Torre y F. G. ${ }^{a}$ Selgas (eds.). Globalización, riesgo, reflexividad. 
Tres temas de la teoría social contemporánea (pp. 249-274). Madrid: CIS.

Renn, 0. (2008). Risk governance: coping with uncertainty in a complex world. London: Earthscan.

Rothstein, B. (2005). Social traps and the problem of trust. Cambridge: Cambridge University Press.

Sabel, C. F. (1993). Studied trust: Building new forms of cooperation in a volatile economy. $\mathrm{Hu}$ man Relations, 46: 1133-1170.

Seligman, A. B. (1998).Trust and Sociability: On the limits of confidence and role expectations. American Journal of Economics and Sociology 57 (4), 391-404.

Simmel, G. (2013). Filosofía del dinero. Madrid: Capitán Swing.

Sjóberg, L. (2001). Limits of knowledge and the limited importance of trust. Risk Analysis, 21 (1), 189-198.

Solá, R., Prades, A., Esplugas, J., Real, M. (2009). Confianza, incertidumbre y percepción social de las tecnologías avanzadas: un estudio de caso. Revista Internacional de Sociología, 67 (1), 161-175

Stivers, R. (1994). The culture of cynicism: American morality in decline. Oxford: Blackwell.

Sztompka, P. (1999). Trust: A Sociological Theory. Cambridge: Cambridge University Press.

Taylor-Gooby, P., Zinn, J. 0. (2006). Current Directions in Risk Research: 'New Developments in Psychology and Sociology'. Risk Analysis, 26 (2), 397-411.

Thuderoz, Ch. (2003). Introduction au propos: la confiance en question, in V. Mangematin and Ch. Thuderoz (dir.). Des mondes de confiance (pp. 19-30). Paris: CNRS.

Tulloch, J., Lupton, D. (2003). Risk and everyday life. London: SAGE.

Turner, J. H. (2007). Human Emotions: A Sociological Theory. Nueva York: Routledge.

Uslaner, E. (2002). The Moral Foundations of Trust. Cambridge: Cambridge University Press.

Walls, J. Pidgeon, N. Weyman, A., Horlick-Jones, T. (2004). Critical trust: understanding lay per- ceptions of health and safety risk regulation. Health, Risk \& Society, 6 (2), 133-150.

Wilkinson, I. (2006). Risk, Vulnerability and Everyday Life. London: Routledge.

Zinn, J. 0. (ed.) (2008). Social theories of risk and uncertainty. Malden, MA: Blackwell.

\section{NOTAS BIOGRÁFICAS}

Javier Callejo Gallego (Madrid, 1960), profesor titular de Sociología en la Facultad de Ciencias Políticas y Sociología de la UNED y miembro de TRANSOC (UCM). Entre las revistas de ciencias sociales en las que ha publicado se encuentran: Revista Española de Investigaciones Sociológicas, Revista Española de Sociología, Revista Internacional de Sociología, Empiria, Papers, Comunicación y Sociedad, Sociológica, Política y Sociedad, British Food Journal, etc. Es autor de monografías como La audiencia activa (1995), Investigar las audiencias (2001), El techo de cristal en el sistema educativo (junto con C. Gómez y Elena Casado, 2004), Nuevos tiempos de trabajo (junto con C. Prieto y R. Ramos, 2008), Adolescencia entre pantallas (junto con Jesús Gutiérrez, 2012).

Ramón Ramos Torre (Madrid, 1949), catedrático de Sociología en la Facultad de Ciencias Políticas y Sociología de la UCM. Ha publicado múltiples artículos en revistas de ciencias sociales (REIS, RIS, Política y Sociedad, Acta sociológica, Sociológica, Time \& Society, etc.) y de debate intelectual (Revista de Occidente, Revista de Libros, Crítica de la Razón Práctica, Kronoscope, etc.). Es autor, colaborador, coautor o editor, de varios libros como Cronos Dividido. La desigualdad en el uso del tiempo entre hombres y mujeres en España. Madrid, 1990; Tiempo y Sociedad (compilador). Madrid 1992; La sociología de Émile Durkheim. Ciencia social, Tiempo, Religión. Madrid 1999; Nuevos tiempos de trabajo (junto con C. Prieto y J. Callejo). Madrid, 2008. Contemporaneidad(es). (junto con J. Gandarilla y G. Valencia). Madrid, 2012. 